\title{
Diffusiophoresis of a colloidal sphere in nonelectrolyte gradients perpendicular to two plane walls
}

\author{
Huan J. Keh*, Yi W. Wan \\ Department of Chemical Engineering, National Taiwan University, Taipei 10617, Taiwan, ROC
}

Received 30 August 2007; received in revised form 7 November 2007; accepted 11 November 2007

Available online 17 November 2007

\begin{abstract}
The problem of the diffusiophoretic motion of a spherical particle in a fluid solution of a nonionic solute situated at an arbitrary position between two infinite parallel plane walls is studied theoretically in the quasisteady limit of negligible Peclet and Reynolds numbers. The applied solute concentration gradient is uniform and perpendicular to the plane walls. The particle-solute interaction layer at the particle surface is assumed to be thin relative to the particle radius and to the particle-wall gap widths, but the polarization effect of the diffuse solute in the thin interfacial layer caused by the strong adsorption of the solute is incorporated. The presence of the walls causes two basic effects on the particle velocity: first, the local solute concentration gradient on the particle surface is altered by the walls, thereby speeding up or slowing down the moving particle; second, the walls enhance the viscous retardation of the particle. A boundary-collocation method is used to semianalytically solve the solutal and hydrodynamic governing equations of the system. Numerical results for the diffusiophoretic velocity of the particle relative to that under identical conditions in an unbounded fluid solution are presented for various cases. The collocation results agree well with the approximate analytical solutions obtained by using a method of reflections. The net effect of the confining walls is always to reduce the particle velocity, irrespective of the surface properties of the particle or the relative particle-wall separation distances. The boundary effect on diffusiophoresis of a particle normal to two plane walls is found to be quite significant and generally stronger than that parallel to the walls. (C) 2007 Elsevier Ltd. All rights reserved.
\end{abstract}

Keywords: Diffusiophoresis; Electrophoresis; Colloidal phenomena; Fluid mechanics; Boundary effect; Plane walls

\section{Introduction}

A colloidal particle, when placed in a solution that is not uniform in the concentration of some molecular solute, will spontaneously migrate toward regions of higher or lower concentration of the solute as a result of physical interaction between the solute molecules and the particle. This migration is termed diffusiophoresis (Dukhin and Derjaguin, 1974) and has been demonstrated experimentally for both ionic (Ebel et al., 1988) and nonionic (Staffeld and Quinn, 1989) solutes. Diffusiophoresis can be utilized to characterize or separate colloidal particles and may be important in some coating processes. In a solution of nonelectrolyte solute with constant concentration gradient $\nabla C_{\infty}$, the diffusiophoretic velocity of a particle

\footnotetext{
* Corresponding author. Fax: +886223623040.

E-mail address: huan@ntu.edu.tw (H.J. Keh).
}

is (Anderson et al., 1982)

$\mathbf{U}^{(0)}=\frac{k T}{\eta} L^{*} K \nabla C_{\infty}$,

and there is no rotational motion of the particle. In the above equation, $L^{*}$ is a characteristic length for the particle-solute interaction (of order $1-10 \mathrm{~nm}$ ), $K$ is the Gibbs adsorption length characterizing the strength of the adsorption of the molecular solute $\left[K\right.$ and $L^{*}$ are defined later by Eqs. (6b) and (6c)], $\eta$ is the fluid viscosity, $k$ is Boltzmann's constant, and $T$ is the absolute temperature. According to Eq. (1), colloidal particles in aqueous solutions with a solute concentration gradient of order $100 \mathrm{kmol} / \mathrm{m}^{4}(=1 \mathrm{M} / \mathrm{cm})$ can move by diffusiophoresis at a velocity of several micrometers per second.

Eq. (1) can be applied to an isolated rigid particle of arbitrary shape and size. However, its validity is based on the assumption that the local radii of curvature of the particle are much larger than the thickness of the particle-solute interaction layer 
(diffuse layer) at the particle surface (of the same order as $L^{*}$ ) and the effect of the polarization of the diffuse solute (the solute continually adsorbs at the upstream edge and desorbs at the downstream edge) in the interfacial layer surrounding the particle is negligible. Anderson and Prieve (1991) analyzed the diffusiophoretic motion of a colloidal sphere of radius $a$ with a thin but polarized diffuse layer when the suspending nonelectrolyte solution is only slightly nonuniform in solute concentration on the length scale of $a\left(a\left|\nabla C_{\infty}\right| \ll C_{\infty}\right)$ and obtained for the particle velocity as

$\mathbf{U}_{0}=A \nabla C_{\infty}$

where the diffusiophoretic mobility

$A=\frac{k T}{\eta} L^{*} K\left(1+\frac{\beta}{a}\right)^{-1}$,

and the definition of the length $\beta$ is given by Eq. (5). For a strongly adsorbing solute (e.g., a surfactant), the relaxation parameter $\beta / a$ (or $K / a$ ) can be much greater than unity. If all the adsorbed solute were "stuck" to the surface of the particle (the diffuseness of the adsorption layer disappears), then $L^{*}=0$ and there would be no diffusiophoretic migration of the particle. In the limit of $\beta / a \rightarrow 0$ (very weak adsorption), the polarization of the diffuse solute in the interfacial layer vanishes and Eq. (2) reduces to Eq. (1). A comparison between Eqs. (1) and (2) indicates that the effect of polarization of the diffuse layer is to decrease the concentration gradient along the particle surface and lower the diffusiophoretic mobility of the particle.

Eq. (2) serves only for fluid solutions that extend to infinity in all directions. In real situations of diffusiophoresis, however, colloidal particles are not isolated and will move in the presence of neighboring boundaries. In the limiting case that Eq. (1) is applicable, the normalized velocity field of the unbounded fluid that is dragged by a particle during diffusiophoresis is the same as for electrophoresis of a dielectric particle with an infinitesimally thin electric double layer (Anderson, 1989); thus, the boundary effects on electrophoresis, which have been investigated extensively in the past (Morrison and Stukel, 1970; Keh and Anderson, 1985; Keh and Chen, 1988; Keh and Lien, 1991; Loewenberg and Davis, 1995; Keh and Chiou, 1996; Hao and Haber, 1998; Yariv and Brenner, 2003; Unni et al., 2007; Hsieh and Keh, 2007), can be utilized to interpret those on diffusiophoresis. An important result of these investigations is that the boundary effects on electrophoresis (and on diffusiophoresis) are weaker than on sedimentation, because the disturbance to the fluid velocity field caused by a phoretic particle decays faster than that produced by a Stokeslet.

When the polarization effect of solute species in the diffuse layer surrounding the particle is considered, the boundary effects on diffusiophoresis can be quite different from those on electrophoresis, due to the fact that the particle size and some other unique factors are involved in each transport mechanism. Through the use of a boundary-collocation technique, the diffusiophoretic (and electrophoretic) motion of a colloidal sphere with a thin but polarized diffuse layer in the direction normal to a plane wall was examined (Keh and Jan, 1996). Recently, the diffusiophoretic motions of a colloidal sphere with a thin polarized diffuse layer parallel to two plane walls at an arbitrary position between them (Chen and Keh, 2002) and along the axis of a circular cylindrical pore (Keh and Hsu, 2006) were also investigated by using the boundary-collocation method. Numerical results of wall-corrections to Eq. (2) for the particle velocity were presented for various values of the relative separation distances and the relaxation parameter $\beta / a$.

This paper is an extension of the previous work (Chen and Keh, 2002) to the situation of the diffusiophoretic motion of a spherical particle with a thin but polarized diffuse layer perpendicular to two parallel plane walls at an arbitrary position between them. The effects of fluid inertia as well as solute convection are neglected. For the case of a particle undergoing diffusiophoresis normal to the plane walls, the diffusion around the particle may generate larger solute concentration gradients on the particle surface relative to those in an infinite medium. These gradients enhance the diffusiophoretic velocity, although their action will be retarded by the viscous interaction of the migrating particle with the confining walls. Both effects of this solutal enhancement and hydrodynamic retardation increase as the ratios of the radius of the particle to its distances from the walls increase. Determining which effect is overriding at small particle-wall gap widths is a main target of this study. Because the governing equations and boundary conditions concerning the general problem of diffusiophoresis of a particle at an arbitrary position between two parallel plane walls in an arbitrary direction are linear, its solution can be obtained as a superposition of the solutions for its two subproblems: motion parallel to the plane walls, which was previously examined (Chen and Keh, 2002), and motion normal to the confining walls, which is considered in this article.

\section{Analysis}

We consider the quasisteady diffusiophoresis of a spherical particle of radius $a$ in a fluid solution of a nonelectrolyte solute perpendicular to two infinite plane walls whose distances from the center of the particle are $b$ and $c$, as shown in Fig. 1. Here $(\rho, \phi, z)$ and $(r, \theta, \phi)$ denote the circular cylindrical and

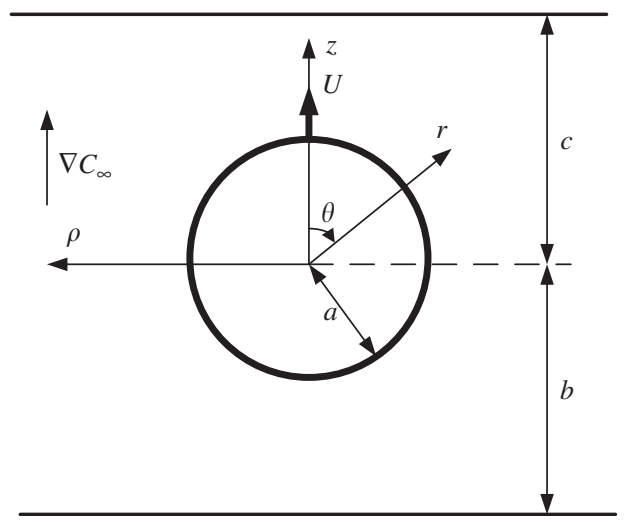

Fig. 1. Geometrical sketch for the diffusiophoresis of a spherical particle perpendicular to two plane walls at an arbitrary position between them. 
spherical coordinate systems, respectively, and the origin of coordinates is chosen at the particle center. A linear solute concentration field $C_{\infty}(z)$ with a uniform gradient $E_{\infty} \mathbf{e}_{z}\left(=\nabla C_{\infty}\right)$ is imposed in the ambient fluid far removed from the particle, where $\mathbf{e}_{z}$ is the unit vector in the $z$ direction and, for convenience, $E_{\infty}$ is taken to be positive. It is assumed that the layer of interaction between the solute molecules and the particle surface is thin in comparison with the radius of the particle and the spacing between the particle and each wall. Hence, the fluid phase can be divided into two regions: an"inner"region defined as the thin interaction layers adjacent to the solid surfaces and an "outer" region defined as the remainder of the fluid phase. The purpose is to obtain the correction to Eq. (2) for the particle mobility due to the presence of the plane walls.

To determine the diffusiophoretic velocity of the particle, it is necessary to ascertain the solute concentration and velocity fields in the fluid phase.

\subsection{Solute concentration distribution}

The diffusiophoretic motion of a particle can be considered quasisteady if the Peclet and Reynolds numbers of the system are small (which is certainly justified for all practical situations since the typical order of magnitude of $U_{0} a$ is $10^{-12} \mathrm{~m}^{2} / \mathrm{s}$ ). The equation of continuity governing the solute concentration distribution $C$ for the outer region of the fluid solution of constant solute diffusivity is the Laplace equation

$\nabla^{2} C=0$.

This governing equation satisfies the boundary condition at the particle "surface" (outer limit of the thin interfacial layer) obtained by solving for the solute concentration in the inner region and using a matching procedure to ensure a continuous solution in the whole fluid phase (O'Brien, 1983; Anderson and Prieve, 1991),

$r=a: \frac{\partial C}{\partial r}=-\beta \frac{1}{r^{2} \sin \theta} \frac{\partial}{\partial \theta}\left(\sin \theta \frac{\partial C}{\partial \theta}\right)$,

in which $\beta$ is the relaxation coefficient defined by

$\beta=(1+v P e) K$,

where

$P e=\frac{k T}{\eta D} L^{*} K C_{0}$,

$K=\int_{0}^{\infty}\left[\exp \left(-\Phi\left(y_{n}\right) / k T\right)-1\right] \mathrm{d} y_{n}$

$L^{*}=K^{-1} \int_{0}^{\infty} y_{n}\left[\exp \left(-\Phi\left(y_{n}\right) / k T\right)-1\right] \mathrm{d} y_{n}$,

and

$v=\left(L^{*} K^{2}\right)^{-1} \int_{0}^{\infty}\left\{\int_{y_{n}}^{\infty}\left[\exp \left(-\Phi\left(y_{n}\right) / k T\right)-1\right] \mathrm{d} y_{n}\right\}^{2} \mathrm{~d} y_{n}$.

In the above equations, $\Phi$ represents the potential energy resulting from the interaction between a single solute molecule and the particle surface; $D$ is the solute diffusion coefficient; $y_{n}$ is the normal distance measured from the particle surface into the fluid phase; $C_{0}$ is the bulk concentration of the solute species measured at the particle center in the absence of the particle. The dimensionless parameter $v$ is of order unity and $v P e$ accounts for the effect of convection on the solute distribution just outside the adsorption layer surrounding the particle. To obtain Eqs. (4)-(6), it was assumed that the concentration of solute within the adsorption boundary layer is related to the solute-surface interaction energy by a Boltzmann distribution.

Since the solute concentration far away from the particle approaches the undisturbed distribution, we can write

$z=c: C=C_{0}+E_{\infty} c$,

$z=-b: C=C_{0}-E_{\infty} b$,

$\rho \rightarrow \infty,-b \leqslant z \leqslant c: C \rightarrow C_{\infty}=C_{0}+E_{\infty} z$.

The concentrations at the two parallel plane walls have been set equal to different constants to allow a uniform gradient in their normal direction far from the particle.

The solute concentration distribution can be expressed as the superposition

$C=C_{0}+E_{\infty} z+C_{w}+C_{p}$.

Here, $C_{w}$ is a separable solution of Eq. (3) in cylindrical coordinates that represents the disturbance produced by the plane walls and is given by a Fourier-Bessel integral

$C_{w}=E_{\infty} \int_{0}^{\infty}\left[X(\omega) \mathrm{e}^{\omega z}+Y(\omega) \mathrm{e}^{-\omega z}\right] \omega J_{0}(\omega \rho) \mathrm{d} \omega$,

where $J_{n}$ is the Bessel function of the first kind of order $n$; $X(\omega)$ and $Y(\omega)$ are unknown functions of the separation variable $\omega$. The last term on the right-hand side of Eq. (10), $C_{p}$ is a separable solution of Eq. (3) in spherical coordinates representing the disturbance generated by the spherical particle and is given by an infinite series in harmonics,

$C_{p}=E_{\infty} \sum_{m=0}^{\infty} R_{m} r^{-m-1} P_{m}(\cos \theta)$

where $P_{m}$ is the Legendre polynomial of order $m$ and $R_{m}$ are unknown constants. Note that a solution for $C$ of the form given by Eqs. (10)-(12) immediately satisfies the boundary condition at infinity in Eq. (9).

Substituting the solute concentration distribution $C$ given by Eqs. (10)-(12) into the boundary conditions in Eqs. (7) and (8) and applying the Hankel transform on the variable $\rho$ lead to a solution for the functions $X(\omega)$ and $Y(\omega)$ in terms of the coefficients $R_{m}$. After the substitution of this solution into Eqs. (10)-(12), $C$ can be expressed as

$C=C_{0}+E_{\infty} z+E_{\infty} \sum_{m=0}^{\infty} R_{m} \delta_{m}^{(1)}(r, \theta)$,

where the function $\delta_{m}^{(1)}(r, \theta)$ is defined by Eq. (B.1) in Appendix $\mathrm{B}$ (in which the integration must be performed numerically). 
Application of the boundary condition given by Eqs. (4)-(13) yields

$$
\begin{aligned}
& \sum_{m=0}^{\infty} R_{m}\left[\left(1-\frac{2 \beta}{a}\right) \delta_{m}^{(2)}(a, \theta)-\frac{\beta}{a} \delta_{m}^{(3)}(a, \theta)\right] \\
& \quad=\left(\frac{2 \beta}{a}-1\right) \cos \theta,
\end{aligned}
$$

where the definitions of the functions $\delta_{m}^{(2)}(r, \theta)\left[=\partial \delta_{m}^{(1)} / \partial r\right]$ and $\delta_{m}^{(3)}(r, \theta)$ are given by Eqs. (B.2) and (B.3).

To satisfy the conditions in Eq. (14) exactly along the entire surface of the particle would require the solution of the entire infinite array of unknown constants $R_{m}$. However, the collocation method (Ganatos et al., 1980; Keh and Jan, 1996) enforces the boundary condition at a finite number of discrete points on any semicircular longitudinal generating arc of the sphere (from $\theta=0$ to $\pi$ ) and truncates the infinite series in Eq. (13) into a finite one. If the spherical boundary is approximated by satisfying the condition of Eq. (4) at $M$ discrete points on the generating arc, the infinite series in Eq. (13) is truncated after $M$ terms, resulting in a system of $M$ simultaneous linear algebraic equations in the truncated form of Eq. (14). This matrix equation can be numerically solved to yield the $M$ unknown constants $R_{m}$ required in the truncated form of Eq. (13) for the solute concentration distribution. The accuracy of the boundary-collocation/truncation technique can be improved to any degree by taking a sufficiently large value of $M$. Naturally, as $M \rightarrow \infty$ the truncation error vanishes and the overall accuracy of the solution depends only on the numerical integration required in evaluating the functions $\delta_{m}^{(2)}$ and $\delta_{m}^{(3)}$ in Eq. (14).

\subsection{Fluid velocity distribution}

Having obtained the solution for the solute concentration distribution on the particle surface which drives the diffusiophoretic migration, we can now proceed to find the flow field. Owing to the low Reynolds number encountered in diffusiophoresis, the fluid motion is governed by the quasisteady fourthorder differential equation for viscous axisymmetric creeping flows

$E^{2}\left(E^{2} \Psi\right)=0$

in which the Stokes stream function $\Psi$ is related to the components of fluid velocity $\mathbf{v}$ in cylindrical coordinates by $\left(v_{\phi}=0\right)$

$v_{\rho}=\frac{1}{\rho} \frac{\partial \Psi}{\partial z}$

$v_{z}=-\frac{1}{\rho} \frac{\partial \Psi}{\partial \rho}$,

and the Stokes operator $E^{2}$ has the form

$E^{2}=\rho \frac{\partial}{\partial \rho}\left(\frac{1}{\rho} \frac{\partial}{\partial \rho}\right)+\frac{\partial^{2}}{\partial z^{2}}$.
The boundary conditions for the fluid velocity at the particle surface, on the plane walls, and far from the particle are

$r=a: \quad \mathbf{v}=U \mathbf{e}_{z}-\frac{k T}{\eta} L^{*} K \frac{\partial C}{r \partial \theta} \mathbf{e}_{\theta}$,

$z=c,-b: \mathbf{v}=\mathbf{0}$,

$\rho \rightarrow \infty,-b \leqslant z \leqslant c: \mathbf{v}=\mathbf{0}$,

where $\mathbf{e}_{\theta}$ is the unit vector in the $\theta$ direction, $U$ is the diffusiophoretic velocity of the particle to be determined, and $\partial C / \partial \theta$ at the particle surface is obtained from the concentration distribution given by Eq. (13).

To solve the fluid flow field, we express the stream function in the form

$\Psi=\Psi_{w}+\Psi_{p}$

Here $\Psi_{w}$ is a Fourier-Bessel integral solution of Eq. (15) in cylindrical coordinates that represents the disturbance produced by the plane walls and is given by (Ganatos et al., 1980)

$$
\begin{aligned}
\Psi_{w}= & \int_{0}^{\infty}\left[A(\omega) \mathrm{e}^{\omega z}+B(\omega) \mathrm{e}^{-\omega z}+C(\omega) \omega z \mathrm{e}^{\omega z}\right. \\
& \left.+D(\omega) \omega z \mathrm{e}^{-\omega z}\right] \rho J_{1}(\omega \rho) \mathrm{d} \omega
\end{aligned}
$$

where $A(\omega), B(\omega), C(\omega)$, and $D(\omega)$ are unknown functions of $\omega$. The second part of $\Psi$, denoted by $\Psi_{p}$, is a separable solution of Eq. (15) in spherical coordinates representing the disturbance generated by the spherical particle and is given by

$\Psi_{p}=\sum_{n=2}^{\infty}\left(B_{n} r^{-n+1}+D_{n} r^{-n+3}\right) G_{n}^{-1 / 2}(\cos \theta)$,

where $G_{n}^{-1 / 2}$ is the Gegenbauer polynomial of the first kind of order $n$ and degree $-\frac{1}{2} ; B_{n}$ and $D_{n}$ are unknown constants. Note that the boundary condition in Eq. (20) is immediately satisfied by a solution of the form given by Eqs. (21)-(23).

Substituting the stream function $\Psi$ given by Eqs. (21)-(23) into the boundary conditions in Eq. (19) and applying the Hankel transform on the variable $\rho$ lead to a solution for $A(\omega)$, $B(\omega), C(\omega)$, and $D(\omega)$ in terms of the coefficients $B_{n}$ and $D_{n}$. After the substitution of this solution into Eqs. (21)-(23), the fluid velocity components can be expressed as

$v_{\rho}=\sum_{n=2}^{\infty}\left[B_{n} \gamma_{1 n}^{(1)}(r, \theta)+D_{n} \gamma_{2 n}^{(1)}(r, \theta)\right]$,

$v_{z}=\sum_{n=2}^{\infty}\left[B_{n} \gamma_{1 n}^{(2)}(r, \theta)+D_{n} \gamma_{2 n}^{(2)}(r, \theta)\right]$

where the definitions of the functions $\gamma_{i n}^{(j)}$ for $i$ and $j$ equal to 1 or 2 are given by Eqs. (B.5) and (B.6) in Appendix B (in which the integration must be performed numerically).

The only boundary condition that remains to be satisfied is that on the particle surface. Substituting Eqs. (13) and (24) 
into Eq. (18), one obtains

$$
\begin{aligned}
& \sum_{n=2}^{\infty}\left[B_{n} \gamma_{1 n}^{(1)}(a, \theta)+D_{n} \gamma_{2 n}^{(1)}(a, \theta)\right] \\
& \quad=U_{0}\left(1+\frac{\beta}{a}\right)\left[\sin \theta-\sum_{m=1}^{\infty} R_{m} \delta_{m}^{(4)}(a, \theta)\right] \cos \theta, \\
& \sum_{n=2}^{\infty}\left[B_{n} \gamma_{1 n}^{(2)}(a, \theta)+D_{n} \gamma_{2 n}^{(2)}(a, \theta)\right] \\
& \quad=U-U_{0}\left(1+\frac{\beta}{a}\right)\left[\sin \theta-\sum_{m=1}^{\infty} R_{m} \delta_{m}^{(4)}(a, \theta)\right] \sin \theta
\end{aligned}
$$

where $U_{0}$ is the diffusiophoretic velocity of the particle in the absence of the plane walls given by Eq. (2) and the definition of the function $\delta_{m}^{(4)}(r, \theta)\left[=\delta_{m}^{(1)} / r \partial \theta\right]$ is given by Eq. (B.4). The first $M$ coefficients $R_{m}$ have been determined through the procedure given in the previous subsection.

Eq. (25) can be satisfied by utilizing the boundary-collocation technique presented for the solution of the solute concentration field. Along a longitudinal generating arc at the particle surface, Eq. (25) is applied at $N$ discrete points (values of $\theta$ between 0 and $\pi$ ) and the infinite series in Eq. (24) are truncated after $N$ terms. This generates a set of $2 N$ linear algebraic equations for the $2 N$ unknown coefficients $B_{n}$ and $D_{n}$. The fluid velocity field is completely obtained once these coefficients are solved for a sufficiently large number of $N$.

\subsection{Derivation of the particle velocity}

The hydrodynamic force acting on the spherical particle can be determined from (Happel and Brenner, 1983)

$F=4 \pi \eta D_{2}$.

This expression shows that only the lowest-order coefficient $D_{2}$ contributes to the drag force exerted on the particle by the fluid.

Since the particle is freely suspended in the surrounding fluid, the net force acting on the particle must vanish. Applying this constraint to Eq. (26), one has

$D_{2}=0$.

To determine the diffusiophoretic velocity $U$ of the particle, Eq. (27) and the $2 N$ algebraic equations resulting from the truncated form of Eq. (25) are to be solved simultaneously. Because of the linearity of the problem, if the sign of the solute concentration gradient is inverted, that of the particle velocity is also inverted. Accordingly, the problem is invariant when the distances $b$ and $c$ from the particle center to the two plane walls are interchanged.

If the particle velocity in Eq. (18) is disabled (i.e., $U=0$ is set), then the force obtained from Eq. (26) can be taken as the diffusiophoretic force exerted on the particle near the walls due to the solute concentration gradient $\nabla C_{\infty}$. This force can be expressed as

$F=6 \pi \eta a U_{0} F^{*}$, where $F^{*}$ is the normalized magnitude of the diffusiophoretic force. The value of $F^{*}$ also equals $f^{*} U / U_{0}$, where $f^{*}$ is the dimensionless Stokes resistance coefficient of the particle (with no slip) migrating normal to the two plane walls driven by a body force in the absence of the solute concentration gradient (Ganatos et al., 1980; Chang and Keh, 2006) and $U$ is the diffusiophoretic velocity of the particle obtained from Eq. (27).

\section{Results and discussion}

The numerical results for the diffusiophoretic motion of a spherical particle perpendicular to two plane walls at an arbitrary position between them, obtained by using the boundary collocation method described in the previous section, are presented in this section. The system of linear algebraic equations to be solved for the coefficients $R_{m}$ is constructed from Eq. (14), while that for $B_{n}$ and $D_{n}$ is composed of Eq. (25). All the numerical integrations to evaluate the functions $\delta_{m}^{(j)}$ and $\gamma_{i n}^{(j)}$ were done by the Gauss-Laguerre quadrature of 180 zeros.

When selecting the points along the half-circular generating arc of the spherical particle where the boundary conditions are to be exactly satisfied, the first points that should be chosen are $\theta=0$ and $\pi$, since these stagnation points control the gaps between the particle and the plane walls. In addition, the point $\theta=\pi / 2$ which defines the projected area of the particle normal to the direction of migration is also important. However, an examination of the systems of linear algebraic equations (14) and (25) shows that the matrix equations become singular if these points are used. To overcome this difficulty, these three points are replaced by four closely adjacent basic points at $\theta=\delta$, $\pi / 2-\delta, \pi / 2+\delta$, and $\pi-\delta$ (Ganatos et al., 1980). Additional points along the generating arc are selected as mirror-image pairs about the equatorial plane $\theta=\pi / 2$ to divide the two quarter-circular arcs of the particle into equal segments. The optimum value of $\delta$ in this work is found to be $0.01^{\circ}$, with which the numerical results of the particle velocity converge satisfactorily.

\subsection{Motion normal to a single plane wall}

Numerical solutions for the normalized diffusiophoretic force $F^{*}$ acting on a spherical particle near a single plane wall (with $c \rightarrow \infty$ ) caused by a normal solute concentration gradient, defined by Eq. (28), for the case of $\beta / a=0$ are given in Table 1 for various values of the spacing parameter $a / b$ at the quasisteady state using the boundary-collocation method. All of these results were obtained by choosing the number of collocation points $N(=M)$ equal to 52,54 , and 56 to show their convergence. The rate of convergence is rapid for small values of $a / b$ and deteriorates as the distance between the particle and the wall decreases. Interestingly, the results in Table 1 illustrate that the diffusiophoretic (or electrophoretic) force exerted on the particle increases monotonically with an increase in the value of $a / b$. For a typical case of a colloidal sphere with radius $a=1 \mu \mathrm{m}$ undergoing diffusiophoresis with velocity $U_{0}$ equal to several $\mu \mathrm{m} / \mathrm{s}$ in aqueous solutions (with 
Table 1

Numerical results of the normalized diffusiophoretic force $F^{*}$ on a spherical particle near a plane wall $(c \rightarrow \infty)$ caused by a normal solute concentration gradient for the case of $\beta / a=0$

\begin{tabular}{|c|c|c|c|}
\hline \multirow[t]{2}{*}{$a / b$} & \multicolumn{3}{|l|}{$F^{*}$} \\
\hline & $N=M=52$ & $N=M=54$ & $N=M=56$ \\
\hline 0.1 & 1.12549 & 1.12549 & 1.12549 \\
\hline 0.2 & 1.27871 & 1.27871 & 1.27871 \\
\hline 0.3 & 1.46357 & 1.46357 & 1.46357 \\
\hline 0.4 & 1.68644 & 1.68644 & 1.68644 \\
\hline 0.5 & 1.95738 & 1.95738 & 1.95738 \\
\hline 0.6 & 2.29213 & 2.29213 & 2.29213 \\
\hline 0.7 & 2.71570 & 2.71570 & 2.71570 \\
\hline 0.8 & 3.26972 & 3.26972 & 3.26972 \\
\hline 0.9 & 4.02842 & 4.02842 & 4.02842 \\
\hline 0.95 & 4.52531 & 4.52531 & 4.52531 \\
\hline 0.99 & 5.00436 & 5.00436 & 5.00436 \\
\hline 0.995 & 5.07048 & 5.07048 & 5.07048 \\
\hline 0.999 & 5.12451 & 5.12451 & 5.12451 \\
\hline
\end{tabular}

a solute concentration gradient of order $100 \mathrm{kmol} / \mathrm{m}^{4}$ ), the diffusiophoretic force acting on the particle given by Eq. (28) ranges from $10^{-13}$ to $10^{-12} \mathrm{~N}$, which is greater than the Brownian force (of order $k T / a$ ) and gravitational/buoyant force exerted on the particle by about two orders of magnitude.

In Table 2, our collocation solutions for the diffusiophoretic velocity of a spherical particle normal to a plane wall for various values of the parameters $\beta / a$ and $a / b$ are presented. The velocity for the diffusiophoretic motion of an identical particle in an infinite fluid, $U_{0}=A E_{\infty}$, given by Eq. (2), is used to normalize the boundary-corrected quantities. Thus, the normalized particle velocity is independent of the value of $k T L^{*} K / \eta$. All of the results obtained under the collocation scheme converge satisfactorily to at least the significant figures shown in the table. Again, the accuracy and convergence behavior of the truncation technique is principally a function of the ratio $a / b$. For general cases with $a / b=0.9$, the numbers of collocation points $M=22$ and $N=22$ can lead to these satisfactory results. For the most difficult case with $a / b=0.999$, the numbers $M=136$ and $N=136$ are sufficiently large to achieve this convergence. Our collocation results in Table 2 can be found to agree excellently with the numerical solutions obtained by Keh and Jan (1996) using a similar boundary-collocation method. Note that the dimensionless Stokes resistance coefficient $f^{*}=F^{*} /\left(U / U_{0}\right)$ for the translation of a rigid sphere normal to a plane wall as a function of $a / b$ can be calculated using the collocation solutions presented in Tables 1 and 2, and the results agree with those available in the literature (Brenner, 1961; Maude, 1961).

In Appendix A, an approximate analytical solution for the same diffusiophoretic motion as that considered here is also obtained by using a method of reflections. The particle velocity is given by Eq. (A.11), which is a power series expansion in $\lambda(=a / b)$. The values of the wall-corrected normalized particle velocity calculated from this asymptotic solution, with the $O\left(\lambda^{9}\right)$ term neglected, are also listed in Table 2 for comparison. It can be seen that the asymptotic formula (A.11) from the method of reflections for $U / U_{0}$ agrees very well with the collocation results as long as $\lambda \leqslant 0.7$; the errors in all cases are less than $3.2 \%$. However, the accuracy of Eq. (A.11) deteriorates rapidly, as expected, when the relative spacing between the particle and the plane wall becomes small (say, $\lambda \geqslant 0.8$ ). The prediction of Eq. (A.11) may overestimate or underestimate the diffusiophoretic velocity of the particle, depending on the combination of the parameters $\beta / a$ and $a / b$.

The collocation solutions for the normalized velocity $U / U_{0}$ of a spherical particle undergoing diffusiophoresis normal to a plane wall as functions of $\beta / a$ are depicted in Fig. 2 for various values of $a / b$. As shown in both Table 1 and Fig. 2, the particle migrates with the velocity that would exist in the absence of the wall, given by Eq. (2), as $a / b \rightarrow 0$. The diffusiophoretic velocity then steadily decreases as the particle approaches the wall (with increasing $a / b$ ), going to zero at the limit. The wall-corrected normalized diffusiophoretic mobility $U / U_{0}$ of the particle increases (or the wall effect is weaker) with an increase in $\beta / a$, keeping $a / b$ unchanged. This increase in the particle mobility in general becomes more pronounced as $a / b$ increases (but is not too close to unity). This behavior is expected knowing that the local solute gradients along the particle surface near a wall with a perpendicularly imposed concentration gradient decrease as $\beta / a$ decreases (the local concentration gradient at the particle surface on the near side to the plane wall is depressed compared with that on the far side, as can be seen in the analysis given in Appendix A or by Keh and Lien, 1991). When $\beta / a=\frac{1}{2}$, the effect of solutal interaction between the particle and the wall disappears, and the relative diffusiophoretic mobility of the particle decreases monotonically with $a / b$ solely owing to the hydrodynamic resistance exerted by the plane wall.

\subsection{Motion perpendicular to two plane walls}

Numerical results of the normalized diffusiophoretic force $F^{*}$ exerted on a spherical particle located between two parallel plane walls whose distance to one wall is the same as to the other (with $c=b$ ) caused by a perpendicular solute concentration gradient are presented in Table 3 for various values of the parameter $a / b$ using the boundary-collocation method for the case of $\beta / a=0$. Like the results for a single plane wall given in Table 1, the results in Table 3 were obtained by choosing the number of collocation points $N(=M)$ equal to 54,56 , and 58 to show their convergence. Again, these results indicate that the diffusiophoretic (or electrophoretic) force acting on the particle is a monotonic increasing function of $a / b$.

In Table 4, the collocation solutions for the normalized velocity $U / U_{0}$ of a spherical particle situated midway between two parallel plane walls (with $c=b$ ) undergoing diffusiophoresis perpendicularly for various values of the parameters $\beta / a$ and $a / b$ are presented. The corresponding method-of-reflection solutions, given by Eq. (A.20) in Appendix A as a power series expansion in $\lambda(=a / b)$ correct to $O\left(\lambda^{8}\right)$, are also listed in this table for comparison. Similar to the case of migration of a spherical particle normal to a single plane wall considered in the previous subsection, the approximate analytical formula 
Table 2

Normalized diffusiophoretic velocity of a spherical particle normal to an infinite plane wall (with $c \rightarrow \infty$ ) computed from the exact boundary-collocation solution and the asymptotic method-of-reflection solution

\begin{tabular}{|c|c|c|c|c|c|c|c|c|}
\hline \multirow[t]{3}{*}{$a / b$} & \multicolumn{8}{|l|}{$U / U_{0}$} \\
\hline & \multicolumn{2}{|l|}{$\beta / a=0$} & \multicolumn{2}{|c|}{$\beta / a=0.1$} & \multicolumn{2}{|l|}{$\beta / a=1$} & \multicolumn{2}{|c|}{$\beta / a=10$} \\
\hline & $\begin{array}{l}\text { Exact } \\
\text { solution }\end{array}$ & $\begin{array}{l}\text { Asymptotic } \\
\text { solution }\end{array}$ & $\begin{array}{l}\text { Exact } \\
\text { solution }\end{array}$ & $\begin{array}{l}\text { Asymptotic } \\
\text { solution }\end{array}$ & $\begin{array}{l}\text { Exact } \\
\text { solution }\end{array}$ & $\begin{array}{l}\text { Asymptotic } \\
\text { solution }\end{array}$ & $\begin{array}{l}\text { Exact } \\
\text { solution }\end{array}$ & $\begin{array}{l}\text { Asymptotic } \\
\text { solution }\end{array}$ \\
\hline 0.1 & 0.99938 & 0.99938 & 0.99941 & 0.99941 & 0.99956 & 0.99956 & 0.99972 & 0.99972 \\
\hline 0.2 & 0.99504 & 0.99505 & 0.99532 & 0.99532 & 0.99655 & 0.99654 & 0.99778 & 0.99777 \\
\hline 0.5 & 0.92089 & 0.92178 & 0.92563 & 0.92607 & 0.94614 & 0.94472 & 0.96636 & 0.96243 \\
\hline 0.6 & 0.85862 & 0.86091 & 0.86739 & 0.86841 & 0.90437 & 0.90014 & 0.94076 & 0.92911 \\
\hline 0.7 & 0.76297 & 0.76854 & 0.77800 & 0.78069 & 0.83997 & 0.83031 & 0.90133 & 0.87298 \\
\hline 0.8 & 0.61631 & 0.63083 & 0.64030 & 0.64960 & 0.73831 & 0.72288 & 0.83840 & 0.78071 \\
\hline 0.9 & 0.38585 & 0.42760 & 0.41854 & 0.45582 & 0.56121 & 0.56023 & 0.72454 & 0.63341 \\
\hline 0.95 & 0.21993 & 0.29290 & 0.25031 & 0.32731 & 0.40497 & 0.45101 & 0.61604 & 0.53159 \\
\hline
\end{tabular}

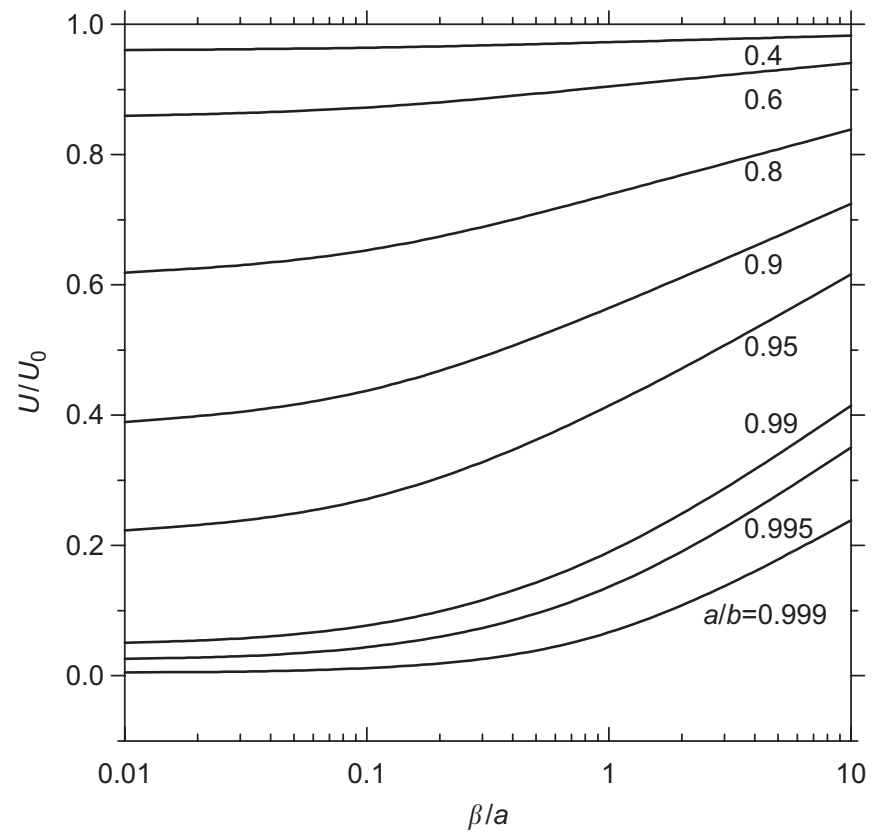

Fig. 2. Plots of the normalized diffusiophoretic velocity $U / U_{0}$ of a spherical particle perpendicular to a plane wall versus the relaxation parameter $\beta / a$ for various values of $a / b$.

(A.20) agrees quite well with the collocation results as long as $\lambda \leqslant 0.6$, but can have significant errors when $\lambda \geqslant 0.7$. In general, Eq. (A.20) overestimates the diffusiophoretic velocity of the particle. A comparison between Table 4 for the case of a slit and Table 2 for the case of a single normal plane indicates that the assumption that the boundary effect for two walls can be obtained by simple addition of single-wall effects leads to a greater correction to diffusiophoretic motion. Analogous to the motion normal to a single plane wall, the dimensionless Stokes resistance coefficient $f^{*}=F^{*} /\left(U / U_{0}\right)$ for the translation of
Table 3

Numerical results of the normalized diffusiophoretic force $F^{*}$ on a spherical particle located midway between two parallel plane walls (with $c=b$ ) caused by a normal solute concentration gradient for the case of $\beta / a=0$

\begin{tabular}{|c|c|c|c|}
\hline \multirow{2}{*}{$a / b$} & \multicolumn{3}{|l|}{$F^{*}$} \\
\hline & $N=M=54$ & $N=M=56$ & $N=M=58$ \\
\hline 0.1 & 1.16746 & 1.16746 & 1.16746 \\
\hline 0.2 & 1.38489 & 1.38489 & 1.38489 \\
\hline 0.3 & 1.66161 & 1.66161 & 1.66161 \\
\hline 0.4 & 2.00976 & 2.00976 & 2.00976 \\
\hline 0.5 & 2.44707 & 2.44707 & 2.44707 \\
\hline 0.6 & 3.00121 & 3.00121 & 3.00121 \\
\hline 0.7 & 3.71724 & 3.71724 & 3.71724 \\
\hline 0.8 & 4.67150 & 4.67150 & 4.67150 \\
\hline 0.9 & 6.00120 & 6.00120 & 6.00120 \\
\hline 0.95 & 6.88224 & 6.88224 & 6.88224 \\
\hline 0.99 & 7.73715 & 7.73715 & 7.73715 \\
\hline 0.995 & 7.85550 & 7.85550 & 7.85550 \\
\hline 0.999 & 7.95228 & 7.95228 & 7.95228 \\
\hline
\end{tabular}

a sphere perpendicular to two plane walls with $c=b$ as a function of $a / b$ can be calculated using the collocation solutions presented in Tables 3 and 4, and the results agree with those available in the literature (Ganatos et al., 1980; Chang and Keh, 2006).

The collocation results for the normalized diffusiophoretic mobility $U / U_{0}$ of a spherical particle on the median plane between two parallel plane walls in the normal direction are plotted in Fig. 3 as a function of $a / b$ for several values of $\beta / a$. Analogous to the corresponding motion of a particle perpendicular to a single plane wall discussed in the previous subsection, whose results are also plotted as dashed curves in this figure for a comparison, $U / U_{0}$ decreases with an increase in $a / b$ for a given value of $\beta / a$ and increases (or the boundary effect becomes weaker) with an increase in $\beta / a$ for a specified value of $a / b$. 
Table 4

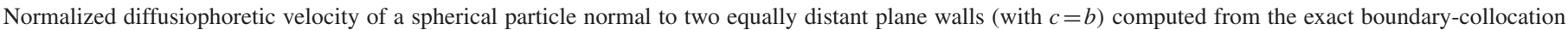
solution and the asymptotic method-of-reflection solution

\begin{tabular}{|c|c|c|c|c|c|c|c|c|}
\hline \multirow[t]{3}{*}{$a / b$} & \multicolumn{8}{|l|}{$U / U_{0}$} \\
\hline & \multicolumn{2}{|l|}{$\beta / a=0$} & \multicolumn{2}{|c|}{$\beta / a=0.1$} & \multicolumn{2}{|l|}{$\beta / a=1$} & \multicolumn{2}{|l|}{$\beta / a=10$} \\
\hline & $\begin{array}{l}\text { Exact } \\
\text { solution }\end{array}$ & $\begin{array}{l}\text { Asymptotic } \\
\text { solution }\end{array}$ & $\begin{array}{l}\text { Exact } \\
\text { solution }\end{array}$ & $\begin{array}{l}\text { Asymptotic } \\
\text { solution }\end{array}$ & $\begin{array}{l}\text { Exact } \\
\text { solution }\end{array}$ & $\begin{array}{l}\text { Asymptotic } \\
\text { solution }\end{array}$ & $\begin{array}{l}\text { Exact } \\
\text { solution }\end{array}$ & $\begin{array}{l}\text { Asymptotic } \\
\text { solution }\end{array}$ \\
\hline 0.1 & 0.99891 & 0.99891 & 0.99900 & 0.99900 & 0.99936 & 0.99936 & 0.99973 & 0.99973 \\
\hline 0.2 & 0.99143 & 0.99143 & 0.99208 & 0.99208 & 0.99501 & 0.99501 & 0.99796 & 0.99796 \\
\hline 0.3 & 0.97174 & 0.97184 & 0.97388 & 0.97398 & 0.98364 & 0.98371 & 0.99359 & 0.99365 \\
\hline 0.4 & 0.93503 & 0.93594 & 0.93996 & 0.94077 & 0.96257 & 0.96318 & 0.98617 & 0.98671 \\
\hline 0.5 & 0.87734 & 0.88200 & 0.88655 & 0.89071 & 0.92934 & 0.93250 & 0.97570 & 0.97853 \\
\hline 0.6 & 0.79470 & 0.81170 & 0.80984 & 0.82501 & 0.88087 & 0.89263 & 0.96220 & 0.97294 \\
\hline 0.7 & 0.68163 & 0.73087 & 0.70433 & 0.74843 & 0.81172 & 0.84705 & 0.94488 & 0.97768 \\
\hline 0.8 & 0.52842 & 0.64971 & 0.55979 & 0.66961 & 0.71000 & 0.80272 & 0.92002 & 1.00715 \\
\hline 0.9 & 0.31579 & 0.58245 & 0.35284 & 0.60073 & 0.54190 & 0.77135 & 0.87079 & 1.08655 \\
\hline 0.95 & 0.17548 & 0.55918 & 0.20753 & 0.57456 & 0.39558 & 0.76596 & 0.80919 & 1.15733 \\
\hline 0.99 & 0.03874 & 0.54749 & 0.05054 & 0.55936 & 0.16699 & 0.76926 & 0.63655 & 1.23529 \\
\hline 0.995 & 0.01966 & 0.54652 & 0.02625 & 0.55787 & 0.11057 & 0.77025 & 0.56154 & 1.24662 \\
\hline 0.999 & 0.0040 & 0.5458 & 0.0055 & 0.5568 & 0.0403 & 0.7712 & 0.4132 & 1.2560 \\
\hline
\end{tabular}

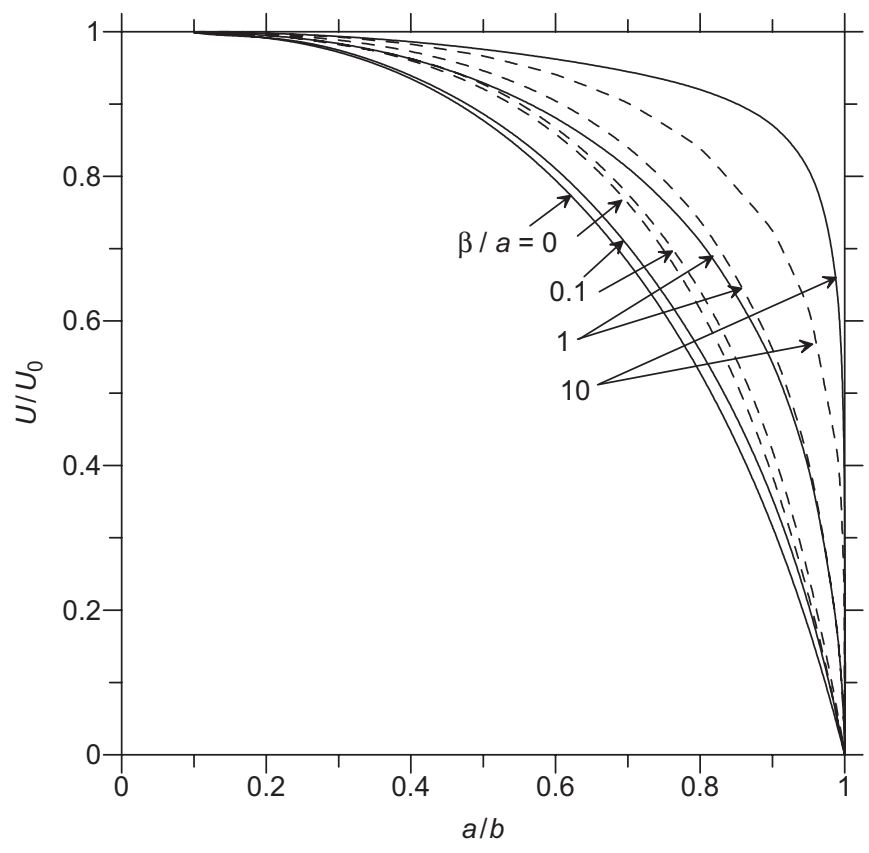

Fig. 3. Plots of the normalized velocity $U / U_{0}$ of a spherical particle situated midway between two parallel plane walls (with $c=b$ ) undergoing diffusiophoresis perpendicularly versus the separation parameter $a / b$ for several values of $\beta / a$. The dashed curves are plotted for the corresponding case of a single plane wall for comparison.

A careful comparison of the curves in Fig. 3 (or of Tables 2 and 4 ) for the case of a slit with the case of a single wall reveals an interesting feature of the boundary effect on diffusiophoresis of a colloidal sphere. The presence of a second normal plane wall, even at a symmetric position with respect to the sphere against the first, does not always enhance the wall effect on the diffusiophoretic particle induced by the first plate only, as indicated in the example case of $\beta / a=10$. This result reflects the fact that the wall can affect the solutal driving force and the viscous drag force on a particle in opposite directions for some cases (with $\beta / a>\frac{1}{2}$, as discussed in Appendix A). Each force is increased in its own direction as the value of $a / b$ turns small, but to a different degree, for the case of lateral diffusiophoretic motion of a particle in a slit relative to that for the case of migration normal to a single plate. Thus, the net effect composed of these two opposite forces for the slit case is not necessarily to enhance that for the case of a single wall.

In Fig. 4, the collocation results for the normalized velocity $U / U_{0}$ of a colloidal sphere with $\beta / a=0$ and $\frac{1}{2}$ undergoing diffusiophoresis normal to two plane walls at various positions between them are plotted. The dashed curves (with $a / b=$ constant) illustrate the effect of the position of the second wall (at $z=c$ ) on the particle velocity for various values of the relative sphere-to-first-wall spacing $b / a$. The solid curves [with $2 a /(b+c)=$ constant], which are symmetric about $b /(b+c)=\frac{1}{2}$ since $b$ and $c$ can be interchanged, indicate the variation of the particle velocity as a function of the sphere position at various values of the relative wall-to-wall spacing $(b+c) / 2 a$. It can be seen that the net wall effect is to reduce the diffusiophoretic mobility $U / U_{0}$ of the particle. At a constant value of $2 a /(b+c)$, the particle in general experiences a minimum viscous drag force and has a greatest velocity when it is located midway between the two walls (with $c=b$ ). The hydrodynamic drag increases and the diffusiophoretic velocity decreases as the particle approaches either of the walls (or the ratio $b /(b+c)$ decreases $)$, with an exception for the case that $\beta / a$ is small, $2 a /(b+c)$ is small, and $b /(b+c)$ is close to $\frac{1}{2}$ [say, $\beta / a=0,2 a /(b+c) \leqslant 0.2$, and $0.48 \leqslant b /(b+c) \leqslant 0.52$, as illustrated in Fig. 4a]. Interestingly, at some specified values of $a / b$ for the diffusiophoretic particle near a first wall, the presence and approach of a second plate can increase the velocity of the particle when it is far from the particle ( $c$ islarge), and then reduce the particle velocity when it is close to the particle 
a

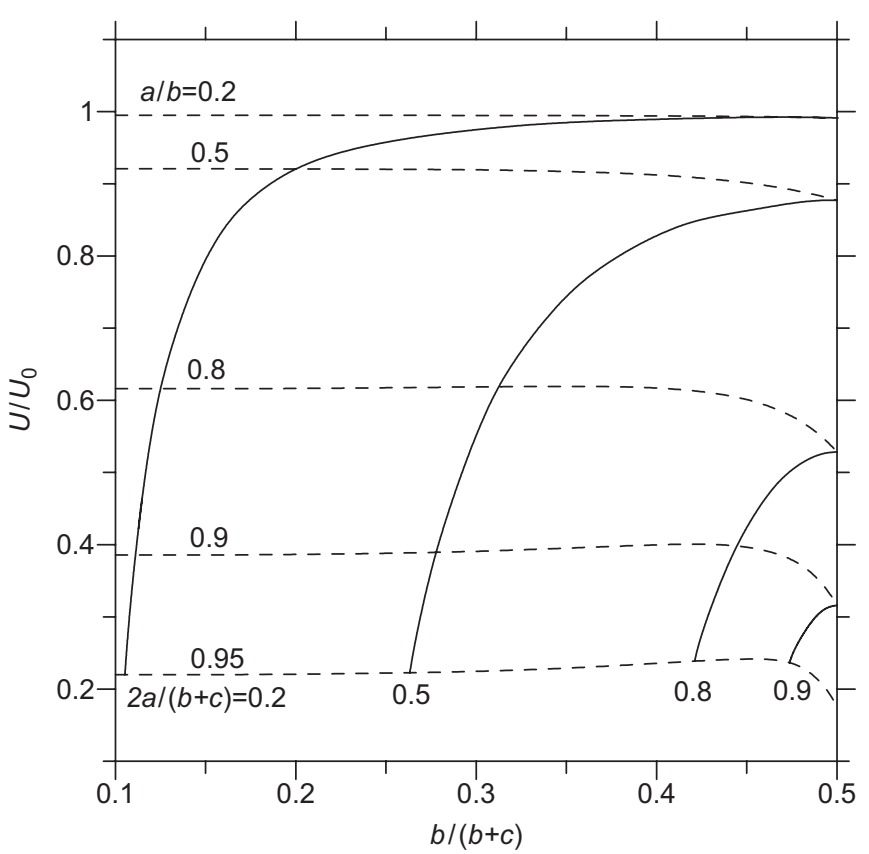

b

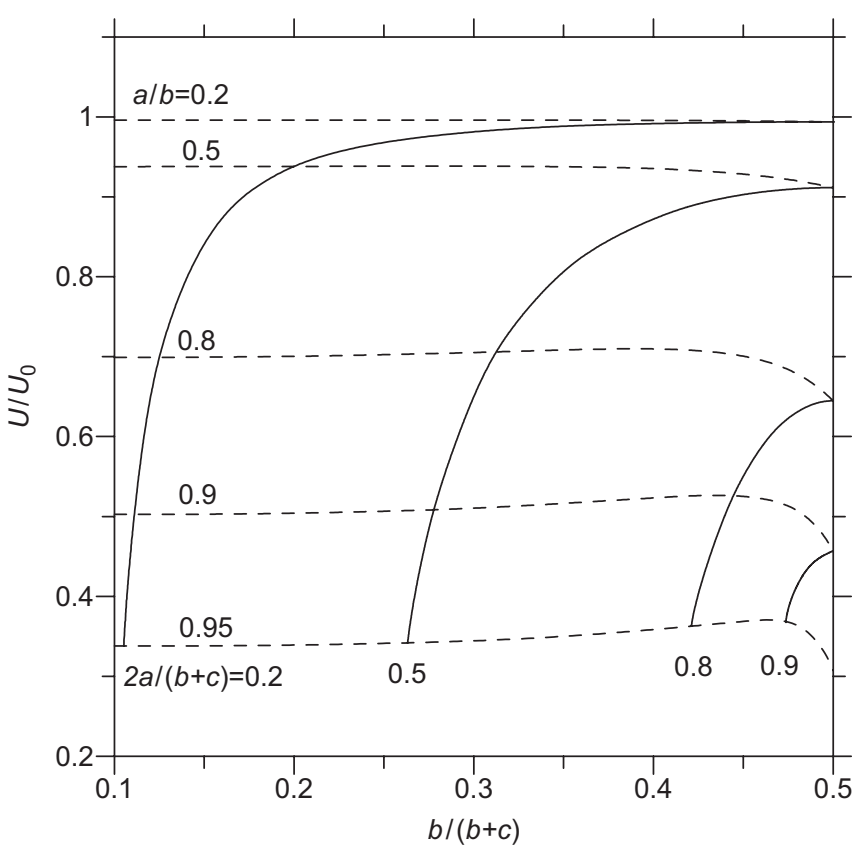

Fig. 4. Plots of the normalized diffusiophoretic velocity $U / U_{0}$ of a spherical particle perpendicular to two plane walls versus the ratio $b /(b+c)$ with $a / b$ and $2 a /(b+c)$ as parameters: (a) $\beta / a=0$; (b) $\beta / a=\frac{1}{2}$.

(say, $b /(b+c)>0.4$ ). Again, Figs. $4 \mathrm{a}$ and $\mathrm{b}$ indicate that the wall effect on the diffusiophoresis of the particle is stronger for the case with a smaller value of $\beta / a$.

For the creeping motion of a spherical particle on which a constant body force $F \mathbf{e}_{z}$ (e.g., a gravitational field) is exerted normal to two infinite plane walls at an arbitrary position between them, the numerical results of the particle velocity have been obtained by using the boundary-collocation technique
(Ganatos et al., 1980; Chang and Keh, 2006). A comparison of our solution with these results indicates that the wall effect on diffusiophoretic motion is weaker than that on a sedimenting particle (also see the discussion after Eq. (A.7) in Appendix A).

Because the governing equations and boundary conditions concerning the general problem of diffusiophoresis of a spherical particle in an arbitrary direction between two parallel plane walls are linear, the net solution can be obtained as a superposition of the solutions for its two subproblems: motion perpendicular to the plane walls, which is examined in this paper, and motion parallel to the confining boundaries. The collocation solutions for the diffusiophoretic motion of a spherical particle parallel to two plane walls have already been obtained by Chen and Keh (2002). It was found that, when the walls are prescribed with a linear solute concentration profile consistent with the far-field concentration distribution, the wallcorrected normalized diffusiophoretic velocity of the particle also increases with an increase in $\beta / a$. A comparison between the Table 3 of Chen and Keh and our Table 4 shows that the plane walls in general exerts the most influence on the particle when diffusiophoretic motion occurs normal to them, and the least in the case of diffusiophoresis parallel to them. Therefore, the direction of diffusiophoretic motion of a particle between two parallel plane walls is different from that of the prescribed solute concentration gradient, except when it is oriented parallel or perpendicular to the plane walls.

\section{Concluding remarks}

The numerical solution and approximate analytical solution for the quasisteady diffusiophoretic motion (and electrophoretic motion, for the case with $\beta / a=0$ ) of a colloidal sphere perpendicular to two infinite plane walls at an arbitrary position between them have been obtained in this work by using the boundary-collocation technique and the method of reflections, respectively, in the limit of vanishingly small Reynolds and Peclet numbers. The boundarycorrected normalized diffusiophoretic velocity $U / U_{0}$ of the particle is found to increase with an increase in $\beta / a$, keeping its distances from the confining walls unchanged. For a specified value of $\beta / a$, the diffusiophoretic mobility of a particle perpendicular to one or two equally distant plane walls is a monotonic decreasing function of the separation parameter $a / b$. The results of the wall-corrected particle mobility reflect the dominance of the hydrodynamic retardation exerted by the confining walls on the particle migration over the possible diffusiophoretic enhancement due to the solutal interaction between the particle and the normal walls. Unfortunately, no experimental data available in the literature involve the diffusiophoretic velocity of a particle as a function of its position between two parallel plane walls or near a single plane wall for a comparison with this theoretical prediction.

The diffusiophoretic mobility of a spherical particle parallel to two infinite plane walls at an arbitrary position between them was calculated in a previous work (Chen and Keh, 2002) for various values of the parameters $\beta / a, a / b$, and $b /(b+c)$. It was found that, for the case of the confining walls prescribed 
with the far-field solute concentration profile under the situation of large $\beta / a$ or for the case of impermeable walls under the situation of small $\beta / a$, the particle mobility first decreases and then increases with increasing $a / b$. When the gaps between the particle and the plane walls turn thin, the particle can even migrate faster than it would as $a / b=0$ (by as much as $22 \%$ for an example case of $c=b$ and $a / b=0.99$ ). The effect of viscous interactions is stronger or the effect of solutal interactions is weaker in a transverse diffusiophoresis than in a parallel motion. In general, the net boundary effect on diffusiophoresis of a particle is stronger for the perpendicular migration. For the general problem of a particle undergoing diffusiophoresis in an arbitrary direction with respect to the two parallel plane walls, the solution can be obtained by adding both the parallel and transverse results vectorially.

\section{Notation}

$a \quad$ radius of the particle, $\mathrm{m}$

A diffusiophoretic mobility defined by Eq. (2), $\mathrm{m}^{5} / \mathrm{s}$

$b, c \quad$ the respective distances from the particle center to the two plates, $\mathrm{m}$

$B \quad$ parameter defined after Eq. (A.8b), $\mathrm{m}^{5} / \mathrm{s}$

$B_{n}, D_{n} \quad$ coefficients in Eq. (23) or (24) for the flow field, $\mathrm{m}^{n+2} / \mathrm{s}, \mathrm{m}^{n} / \mathrm{s}$

$C$ solute concentration field in the fluid, $\mathrm{m}^{-3}$

$C_{0} \quad$ value of $C_{\infty}$ at the position of particle center, $\mathrm{m}^{-3}$

$C_{\infty} \quad$ prescribed solute concentration field defined by Eq. (9), $\mathrm{m}^{-3}$

$d_{1}, d_{2}, d_{3} \quad$ constants defined by Eq. (A.16)

$e_{z}, e_{r}, e_{\theta} \quad$ unit vectors in $z, r$, and $\theta$ directions

$E_{\infty} \quad=\left|\nabla C_{\infty}\right|, \mathrm{m}^{-4}$

$F^{*} \quad$ normalized diffusiophoretic force

$G$

$G_{n}^{-1 / 2}$

H

$J_{n}$

$k$

K

$L^{*}$

$M, N$

$P_{n}$

$r$

$R_{m}$

$T$

$\mathbf{U}, U$

$\mathbf{U}_{0}, U_{0}$

v

$v_{\rho}, v_{z}$

$z$ dimensionless parameter defined after Eq. (A.4b)

Gegenbauer polynomial of the first kind of order $n$ and degree $-\frac{1}{2}$

dimensionless parameter defined after Eq. (A.8b)

Bessel function of the first kind of order $n$

Boltzmann's constant, J/K

Gibbs adsorption length defined by Eq. (6b), m characteristic length for the particle-solute interaction defined by Eq. (6c), m

numbers of collocation points on the particle surface

Legendre function of order $n$

radial spherical coordinate, $\mathrm{m}$

coefficients in Eq. (12) or (13) for the concentration field, $\mathrm{m}^{m+2}$

absolute temperature, $\mathrm{K}$

diffusiophoretic velocity of the particle, $\mathrm{m} / \mathrm{s}$

diffusiophoretic velocity of an isolated particle de-

fined by Eq. (2), m/s

velocity field of the fluid, $\mathrm{m} / \mathrm{s}$

components of $\mathbf{v}$ in cylindrical coordinates, $\mathrm{m} / \mathrm{s}$

axial cylindrical coordinate, $\mathrm{m}$

\section{Greek letters}

$\beta$

$\gamma_{1 n}^{(j)}, \gamma_{2 n}^{(j)}$

relaxation coefficient defined by Eq. (5), m functions of $r$ and $\theta$ defined by Eqs. (B.5) and (B.6), $\mathrm{m}^{-n-1}, \mathrm{~m}^{-n+1}$

$\delta_{n}^{(1)}, \delta_{n}^{(2)}, \delta_{n}^{(3)}, \delta_{n}^{(4)}$ functions of $r$ and $\theta$ defined by Eqs.

(B.1)-(B.4), $\mathrm{m}^{-\mathrm{n}-1}, \mathrm{~m}^{-\mathrm{n}-2}$, $\mathrm{m}^{-\mathrm{n}-2}, \mathrm{~m}^{-\mathrm{n}-2}$

$\eta \quad$ viscosity of the fluid, $\mathrm{kg} / \mathrm{m} \mathrm{s}$

$\theta, \phi \quad$ angular spherical coordinates

$\lambda \quad=a / b$

$\rho \quad$ radial cylindrical coordinate, $\mathrm{m}$

$\Psi \quad$ Stokes stream function for the fluid flow, $\mathrm{m}^{3} / \mathrm{s}$

Subscripts

p particle

Superscript

(i)

the $i$ th reflection

\section{Acknowledgment}

This research was partly supported by the National Science Council of the Republic of China.

\section{Appendix A. Analysis of the diffusiophoresis of a spherical particle normal to one or two plane walls by a method of reflections}

In this appendix, the quasisteady diffusiophoretic motion of a colloidal sphere perpendicular either to an infinite plane wall $(c \rightarrow \infty)$ or to two parallel plane walls with equal distances from the particle $(c=b)$, as shown in Fig. 1, will be analyzed using a method of reflections. The effect of the walls on the particle velocity $\mathbf{U}$ is sought in expansions of $\lambda$, which equals $a / b$, the ratio of the particle radius to the distance between the particle center and the walls.

\section{A.1. Motion normal to a single plane wall}

For the problem of diffusiophoretic motion of a spherical particle normal to an infinite plane wall, the governing equations (3) and (15) must be solved by satisfying the boundary conditions (4), (7)-(9) and (18)-(20) with $c \rightarrow \infty$. The methodof-reflection solution for the solute concentration and velocity fields in the fluid phase consists of the following series, whose terms depend on increasing powers of $\lambda$ :

$$
\begin{aligned}
& C=C_{0}+E_{\infty} z+C_{p}^{(1)}+C_{w}^{(1)}+C_{p}^{(2)}+C_{w}^{(2)}+\cdots, \\
& \mathbf{v}=\mathbf{v}_{p}^{(1)}+\mathbf{v}_{w}^{(1)}+\mathbf{v}_{p}^{(2)}+\mathbf{v}_{w}^{(2)}+\cdots,
\end{aligned}
$$

where subscripts $w$ and $p$ represent the reflections from wall and particle, respectively, and the superscript $(i)$ denotes the $i$ th reflection from that surface. In these series, all the expansion sets of the solute concentration and fluid velocity fields must satisfy Eqs. (3) and (15). 
According to Eq. (A.1), the diffusiophoretic velocity of the particle can also be expressed in the series form

$\mathbf{U}=U_{0} \mathbf{e}_{z}+\mathbf{U}^{(1)}+\mathbf{U}^{(2)}+\cdots$.

In this expression, $U_{0}=A E_{\infty}$ is the diffusiophoretic velocity of an identical particle in the corresponding unbounded solution phase given by Eq. (2); $\mathbf{U}^{(i)}$ is related to $\nabla C_{w}^{(i)}$ and $\mathbf{v}_{w}^{(i)}$ by (Keh and Luo, 1995)

$\mathbf{U}^{(i)}=A\left[\nabla C_{w}^{(i)}\right]_{0}+\left[\mathbf{v}_{w}^{(i)}\right]_{0}+\frac{a^{2}}{6}\left[\nabla^{2} \mathbf{v}_{w}^{(i)}\right]_{0}$,

where the subscript 0 to variables inside brackets denotes evaluation at the position of the particle center.

The solution for the first reflected fields from the particle is

$C_{p}^{(1)}=G E_{\infty} a^{3} r^{-2} \cos \theta$,

$\mathbf{v}_{p}^{(1)}=\frac{1}{2} U_{0} a^{3} r^{-3}\left(2 \cos \theta \mathbf{e}_{r}+\sin \theta \mathbf{e}_{\theta}\right)$,

where $G=\left(\frac{1}{2}-\beta / a\right)(1+\beta / a)^{-1}$. Obviously, $-1 \leqslant G \leqslant \frac{1}{2}$, with the upper and lower bounds occurring at the limits $\beta / a=0$ and $\beta / a \rightarrow \infty$, respectively. The velocity distribution shown in Eq. (A.4b) is identical to the irrotational flow surrounding a rigid sphere moving with velocity $U_{0} \mathbf{e}_{z}$.

The boundary conditions for the $i$ th reflected fields from the wall are derived from Eqs. (7)-(9), (19), and (20),

$$
\begin{aligned}
z=-b: C_{w}^{(i)}=-C_{p}^{(i)}, & \\
\mathbf{v}_{w}^{(i)}=-\mathbf{v}_{p}^{(i)}, & \\
r \rightarrow \infty, z \geqslant-b: C_{w}^{(i)} & \rightarrow 0, \\
\mathbf{v}_{w}^{(i)} & \rightarrow \mathbf{0} .
\end{aligned}
$$

The solution of $C_{w}^{(1)}$ is obtained by applying Hankel transforms on variable $\rho$ in Eqs. (3) and (A.5a), (A.5c) (taking $i=1$ ), with the result

$C_{w}^{(1)}=G E_{\infty} a^{3}(2 b+z)\left[(2 b+z)^{2}+\rho^{2}\right]^{-3 / 2}$.

This reflected concentration field may be interpreted as arising from the reflection of the imposed field $E_{\infty} \mathbf{e}_{z}$ from a fictitious particle identical to the actual particle, its location being at the mirror-image position of the actual particle with respect to the plane $z=-b$ (i.e., at $x=0, y=0, z=-2 b$ ). The solution of $\mathbf{v}_{w}^{(1)}$ can also be obtained by applying Hankel transforms to the Stokes equation (15) twice and to boundary conditions (A.5b), (A.5d), which results in

$$
\begin{aligned}
\mathbf{v}_{w}^{(1)}= & -\frac{3}{2} U_{0} a^{3}\left\{\rho(4 b+3 z)\left[(2 b+z)^{2}+\rho^{2}\right]^{-5 / 2}\right. \\
& \left.-10 \rho(b+z)(2 b+z)^{2}\left[(2 b+z)^{2}+\rho^{2}\right]^{-7 / 2}\right\} \mathbf{e}_{\rho} \\
& -\frac{1}{2} U_{0} a^{3}\left\{2\left[(2 b+z)^{2}+\rho^{2}\right]^{-3 / 2}\right. \\
& +3\left[4(b+z)(2 b+z)-\rho^{2}\right]\left[(2 b+z)^{2}+\rho^{2}\right]^{-5 / 2} \\
& \left.-30 \rho^{2}(b+z)(2 b+z)\left[(2 b+z)^{2}+\rho^{2}\right]^{-7 / 2}\right\} \mathbf{e}_{z} .
\end{aligned}
$$

The contributions of $C_{w}^{(1)}$ and $\mathbf{v}_{w}^{(1)}$ to the velocity of the particle are determined by using Eq. (A.3):

$\mathbf{U}_{s}^{(1)}=A\left[\nabla C_{w}^{(1)}\right]_{r=0}=-\frac{1}{4} G \lambda^{3} U_{0} \mathbf{e}_{z}$,

$$
\mathbf{U}_{h}^{(1)}=\left[\mathbf{v}_{w}^{(1)}+\frac{a^{2}}{6} \nabla^{2} \mathbf{v}_{w}^{(1)}\right]_{r=0}=\left(-\frac{1}{2} \lambda^{3}+\frac{1}{4} \lambda^{5}\right) U_{0} \mathbf{e}_{z},
$$

$$
\mathbf{U}^{(1)}=\mathbf{U}_{s}^{(1)}+\mathbf{U}_{h}^{(1)}=\left[-\left(\frac{1}{2}+\frac{1}{4} G\right) \lambda^{3}+\frac{1}{4} \lambda^{5}\right] U_{0} \mathbf{e}_{z} .
$$

Eq. (A.7a) shows that the reflected solute concentration field from the plane wall can decrease (if $G>0$ or $\beta / a<\frac{1}{2}$ ) or increase (if $G<0$ or $\beta / a>\frac{1}{2}$ ) the diffusiophoretic velocity of the particle, while Eq. (A.7b) indicates that the reflected velocity field is to decrease this velocity; the net effect of the reflected fields is expressed by Eq. (A.7c), which always retards the movement of the particle, irrespective of the combination of the values of $G$ (or $\beta / a$ ) and $\lambda$. When $G=0$ (or $\beta / a=\frac{1}{2}$ ), the reflected concentration field makes no contribution to the diffusiophoretic velocity. Eq. (A.7c) shows that the wall correction to the diffusiophoretic velocity of the particle is $O\left(\lambda^{3}\right)$, which is weaker than that obtained for the corresponding sedimentation problem, in which the leading boundary effect is $O(\lambda)$.

The solution for the second reflected fields from the particle is

$$
\begin{aligned}
C_{p}^{(2)}= & E_{\infty}\left[-\frac{1}{4} G^{2} \lambda^{3} a^{3} r^{-2} \cos \theta\right. \\
& \left.+\frac{3}{16} G H \lambda^{4} a^{4} r^{-3}\left(3 \cos ^{2} \theta-1\right)+O\left(\lambda^{5} a^{5}\right)\right], \\
\mathbf{v}_{p}^{(2)}= & U_{0}\left[-\frac{1}{8} G \lambda^{3} a^{3} r^{-3}\left(2 \cos \theta \mathbf{e}_{r}+\sin \theta \mathbf{e}_{\theta}\right)\right. \\
& -\frac{3}{64}\left(2 G \frac{B}{A}+15\right) \lambda^{4} a^{2} r^{-2}\left(3 \cos ^{2} \theta-1\right) \mathbf{e}_{r} \\
& \left.+O\left(\lambda^{4} a^{4}, \lambda^{5} a^{5}\right)\right],
\end{aligned}
$$

where $H=3(1-2 \beta / a)(3+4 \beta / a)$ and $B=-(5 k T / 6 \eta) L^{*} K(1+$ $2 \beta / a)^{-1}$.

The boundary conditions for the second reflected fields from the wall are obtained by substituting the results of $C_{p}^{(2)}$ and $\mathbf{v}_{p}^{(2)}$ into Eq. (A.5), with which Eqs. (3) and (15) can be solved as before to yield

$$
\begin{aligned}
& {\left[\nabla C_{w}^{(2)}\right]_{r=0}=E_{\infty}\left[-\frac{1}{16} G^{2} \lambda^{6}+O\left(\lambda^{9}\right)\right] \mathbf{e}_{z},} \\
& {\left[\mathbf{v}_{w}^{(2)}+\frac{a^{2}}{6} \nabla^{2} \mathbf{v}_{w}^{(2)}\right]_{r=0}} \\
& \quad=U_{0}\left\{-\frac{1}{256}\left[135-2\left(16+9 \frac{B}{A}\right) G\right] \lambda^{6}\right. \\
& \left.\quad-\frac{1}{16}\left[G+\frac{3}{64}\left(2 G \frac{B}{A}-15\right)\right] \lambda^{8}+O\left(\lambda^{9}\right)\right\} \mathbf{e}_{z} .
\end{aligned}
$$

The contribution of the second reflected fields to the particle velocity is obtained by combining Eqs. (A.3) and (A.9), which gives

$$
\begin{aligned}
\mathbf{U}^{(2)}= & U_{0}\left\{-\frac{1}{256}\left[135-2\left(16+9 \frac{B}{A}\right) G+16 G^{2}\right] \lambda^{6}\right. \\
& \left.-\frac{1}{16}\left[G+\frac{3}{64}\left(2 G \frac{B}{A}-15\right)\right] \lambda^{8}+O\left(\lambda^{9}\right)\right\} \mathbf{e}_{z} .
\end{aligned}
$$


Obviously, $\mathbf{U}^{(4)}$ will be $O\left(\lambda^{9}\right)$. With the substitution of Eqs. (A.7c) and (A.10) into Eq. (A.2), the particle velocity can be expressed as $\mathbf{U}=U \mathrm{e}_{z}$ with

$$
\begin{aligned}
U= & U_{0}\left\{1-\left(\frac{1}{2}+\frac{1}{4} G\right) \lambda^{3}+\frac{1}{4} \lambda^{5}\right. \\
& -\frac{1}{256}\left[135-2\left(16+9 \frac{B}{A}\right) G+16 G^{2}\right] \lambda^{6} \\
& \left.-\frac{1}{16}\left[G+\frac{3}{64}\left(2 G \frac{B}{A}-15\right)\right] \lambda^{8}+O\left(\lambda^{9}\right)\right\} .
\end{aligned}
$$

Owing to the linearity of the problem, the above analysis is valid when the particle is either approaching the plane wall or receding from it.

\section{A.2. Motion normal to two parallel plane walls}

For the problem of diffusiophoretic motion of a colloidal sphere perpendicular to two infinite plane walls with equal distances from the particle, the boundary conditions corresponding to governing Eqs. (3) and (15) are given by Eqs. (4), (7)-(9), and (18)-(20) with $c=b$. With $\lambda=a / b \ll 1$, the series expansions of the solute concentration, fluid velocity, and particle velocity given by Eqs. (A.1), (A.2), and (A.4) remain valid here. From Eqs. (7)-(9), (19), and (20), the boundary conditions for $C_{w}^{(i)}$ and $\mathbf{v}_{w}^{(i)}$ are found to be

$$
\begin{array}{r}
|z|=b: C_{w}^{(i)}=-C_{p}^{(i)}, \\
\mathbf{v}_{w}^{(i)}=-\mathbf{v}_{p}^{(i)}, \\
\rho \rightarrow \infty, z \leqslant|b|: C_{w}^{(i)} \rightarrow 0, \\
\mathbf{v}_{w}^{(i)} \rightarrow \mathbf{0} .
\end{array}
$$

The first wall-reflected fields can be solved by the same method as used for the case of a single plane wall in the previous subsection, with the result

$$
\begin{aligned}
C_{w}^{(1)}= & -G E_{\infty} a \lambda^{2} \int_{0}^{\infty} \frac{1+\mathrm{e}^{-2 \alpha}}{\sinh (2 \alpha)} \sinh \left(\frac{\alpha}{b} z\right) \alpha J_{0}\left(\frac{\alpha}{b} \rho\right) \mathrm{d} \alpha, \\
\mathbf{v}_{w}^{(1)}= & -U_{0} \lambda^{3} \int_{0}^{\infty} \alpha^{2}\left[E(\alpha, z) J_{1}\left(\frac{\alpha}{b} \rho\right) \mathbf{e}_{\rho}\right. \\
& \left.+F(\alpha, z) J_{0}\left(\frac{\alpha}{b} \rho\right) \mathbf{e}_{z}\right] \mathrm{d} \alpha
\end{aligned}
$$

where

$$
\begin{aligned}
E(\alpha, z)= & \frac{2}{2 \alpha+\sinh (2 \alpha)}\left[\left(1-\alpha-\mathrm{e}^{-\alpha} \sinh \alpha\right) \sinh \left(\frac{\alpha}{b} z\right)\right. \\
& \left.+\frac{\alpha}{b} z \cosh \left(\frac{\alpha}{b} z\right)\right], \\
F(\alpha, z)= & \frac{2}{2 \alpha+\sinh (2 \alpha)}\left[\left(\alpha+\mathrm{e}^{-\alpha} \sinh \alpha\right) \cosh \left(\frac{\alpha}{b} z\right)\right. \\
& \left.-\frac{\alpha}{b} z \sinh \left(\frac{\alpha}{b} z\right)\right] .
\end{aligned}
$$

The contributions of $C_{w}^{(1)}$ and $\mathbf{v}_{w}^{(1)}$ to the particle velocity are determined by using Eq. (A.3), which lead to a result similar to Eq. (A.7),

$$
\begin{aligned}
& \mathbf{U}_{t}^{(1)}=A\left[\nabla C_{w}^{(1)}\right]_{r=0}=-d_{1} G \lambda^{3} U_{0} \mathbf{e}_{z}, \\
& \mathbf{U}_{h}^{(1)}=\left[\mathbf{v}_{w}^{(1)}+\frac{a^{2}}{6} \nabla^{2} \mathbf{v}_{w}^{(1)}\right]_{r=0}=\left[-d_{2} \lambda^{3}+d_{3} \lambda^{5}\right] U_{0} \mathbf{e}_{z}, \\
& \mathbf{U}^{(1)}=\mathbf{U}_{t}^{(1)}+\mathbf{U}_{h}^{(1)}=\left[-\left(d_{1} G+d_{2}\right) \lambda^{3}+d_{3} \lambda^{5}\right] U_{0} \mathbf{e}_{z},
\end{aligned}
$$

where

$$
\begin{aligned}
& d_{1}=\int_{0}^{\infty} \frac{1+\mathrm{e}^{-2 \alpha}}{\sinh (2 \alpha)} \alpha^{2} \mathrm{~d} \alpha=0.60103, \\
& d_{2}=\int_{0}^{\infty} \frac{\sinh (\alpha) \mathrm{e}^{-\alpha}+\alpha}{2 \alpha+\sinh (2 \alpha)} \alpha^{2} \mathrm{~d} \alpha=0.79077, \\
& d_{3}=\frac{1}{3} \int_{0}^{\infty} \frac{\alpha^{4}}{2 \alpha+\sinh (2 \alpha)} \mathrm{d} \alpha=0.44176 .
\end{aligned}
$$

Again, Eqs. (A.15a) and (A.16a) show that the reflected concentration field from the confining walls can decrease (if $G>0$ or $\beta / a<\frac{1}{2}$ ) or increase (if $G<0$ or $\beta / a>\frac{1}{2}$ ) the particle velocity, while Eqs. (A.15b) and (A.16b), (A.16c) indicate that the reflected velocity field is to decrease this velocity; the net effect is expressed by Eq. (A.15c), which can enhance or retard the movement of the particle, depending on the combination of the values of $G$ (or $\beta / a$ ) and $\lambda$. Eq. (A.15c) indicates that the necessary condition for the wall enhancement on the diffusiophoretic motion to occur is a large value of $\beta / a$ and a value of $\lambda$ close to unity such that the relation $d_{3} \lambda^{5}>\left(d_{2}+d_{1} G\right) \lambda^{3}$ is warranted.

Analogous to the previous case, the results of the second reflections can be obtained as

$$
C_{p}^{(2)}=-E_{\infty}\left[d_{1} G^{2} \lambda^{3} a^{3} r^{-2} \cos \theta+O\left(\lambda^{5} a^{5}\right)\right],
$$

$\mathbf{v}_{p}^{(2)}=-U_{0} d_{1} G \lambda^{3} a^{3} r^{-3}\left(2 \cos \theta \mathbf{e}_{r}+\sin \theta \mathbf{e}_{\theta}\right)+O\left(\lambda^{5} a^{3}\right)$,

$$
\begin{aligned}
& {\left[\nabla C_{w}^{(2)}\right]_{r=0}=E_{\infty}\left[d_{1}^{2} G^{2} \lambda^{6}+O\left(\lambda^{9}\right)\right] \mathbf{e}_{z},} \\
& {\left[\nabla \mathbf{v}_{w}^{(2)}+\frac{a^{2}}{6} \nabla^{2} \mathbf{v}_{w}^{(2)}\right]_{r=0}} \\
& \quad=U_{0}\left[d_{1} d_{2} G \lambda^{6}-d_{1} d_{3} G \lambda^{8}+O\left(\lambda^{9}\right)\right] \mathbf{e}_{z},
\end{aligned}
$$

and

$\mathrm{U}^{(2)}=\left[\left(d_{1}^{2} G^{2}+d_{1} d_{2} G\right) \lambda^{6}-d_{1} d_{3} G \lambda^{8}+O\left(\lambda^{9}\right)\right] U_{0} \mathbf{e}_{z}$.

Note that the $\lambda^{4} a^{2}$ and $\lambda^{4} a^{4}$ terms in the expressions for $C_{p}^{(2)}$ and $\mathbf{v}_{p}^{(2)}$ vanish.

With the combination of Eqs. (A.2), (A.15c), and (A.19), the particle velocity can be expressed as $\mathbf{U}=U \mathbf{e}_{z}$ with

$$
\begin{aligned}
U= & U_{0}\left[1-\left(d_{2}+d_{1} G\right) \lambda^{3}+d_{3} \lambda^{5}\right. \\
& \left.+\left(d_{1}^{2} G^{2}+d_{1} d_{2} G\right) \lambda^{6}-d_{1} d_{3} G \lambda^{8}+O\left(\lambda^{9}\right)\right] .
\end{aligned}
$$

This result is valid for a particle undergoing diffusiophoresis toward either of the two plane walls. 


\section{Appendix B. Definitions of some functions in Section 2}

The functions $\delta_{m}^{(j)}$ for $j$ equal to 1, 2, 3, or 4 in Eqs. (13), (14), and (25) are defined by

$$
\begin{aligned}
\delta_{m}^{(1)}(r, \theta)= & \int_{0}^{\infty} \omega(\sinh \tau)^{-1}\left[-B_{1 m}^{\prime \prime}(\omega,-b) \sinh \eta\right. \\
& \left.+B_{1 m}^{\prime \prime}(\omega, c) \sinh \sigma\right] J_{0}(r \omega \sin \theta) \mathrm{d} \omega \\
& +r^{-m-1} P_{m}(\cos \theta),
\end{aligned}
$$

$$
\begin{aligned}
\delta_{m}^{(2)}(r, \theta) & \\
= & \int_{0}^{\infty} \omega^{2}(\sinh \tau)^{-1}\left\{\operatorname { s i n } \theta \left[B_{1 m}^{\prime \prime}(\omega,-b) \sinh \eta\right.\right. \\
& \left.-B_{1 m}^{\prime \prime}(\omega, c) \sinh \sigma\right] J_{1}(r \omega \sin \theta) \\
& -\cos \theta\left[B_{1 m}^{\prime \prime}(\omega,-b) \cosh \eta\right. \\
& \left.\left.-B_{1 m}^{\prime \prime}(\omega, c) \cosh \sigma\right] J_{0}(r \omega \sin \theta)\right\} \mathrm{d} \omega \\
& -(m+1) r^{-m-2} P_{m}(\cos \theta),
\end{aligned}
$$

$$
\begin{aligned}
\delta_{m}^{(3)}(r, \theta) & \int_{0}^{\infty} r \omega^{3}(\sinh \tau)^{-1}\left\{\operatorname { s i n } 2 \theta \left[B_{1 m}^{\prime \prime}(\omega,-b) \cosh \eta\right.\right. \\
& \left.-B_{1 m}^{\prime \prime}(\omega, c) \cosh \sigma\right] J_{1}(r \omega \sin \theta) \\
& -\frac{1}{4}\left[B_{1 m}^{\prime \prime}(\omega,-b) \sinh \eta-B_{1 m}^{\prime \prime}(\omega, c) \sinh \sigma\right] \\
& \times\left[(1+3 \cos 2 \theta) J_{0}(r \omega \sin \theta)\right. \\
& \left.\left.+2 \sin ^{2} \theta J_{2}(r \omega \sin \theta)\right]\right\} \mathrm{d} \omega \\
& +(m+1)(m+2) r^{-m-2} P_{m}(\cos \theta),
\end{aligned}
$$

$$
\begin{aligned}
\delta_{m}^{(4)} & (r, \theta) \\
= & \int_{0}^{\infty} \omega^{2}(\sinh \tau)^{-1}\left\{\operatorname { c o s } \theta \left[B_{1 m}^{\prime \prime}(\omega,-b) \sinh \eta\right.\right. \\
& \left.-B_{1 m}^{\prime \prime}(\omega, c) \sinh \sigma\right] J_{1}(r \omega \sin \theta) \\
& +\sin \theta\left[B_{1 m}^{\prime \prime}(\omega,-b) \cosh \eta\right. \\
& \left.\left.-B_{1 m}^{\prime \prime}(\omega, c) \cosh \sigma\right] J_{0}(r \omega \sin \theta)\right\} \mathrm{d} \omega \\
& -(1+m) r^{-m-2}\left[P_{m}(\cos \theta) \cos \theta\right. \\
& \left.-P_{m+1}(\cos \theta)\right] \csc \theta,
\end{aligned}
$$

and the functions $\gamma_{i n}^{(j)}$ for $i$ and $j$ equal to 1 or 2 in Eqs. (24) and (25) are defined by

$$
\begin{aligned}
\gamma_{i n}^{(1)}(r, \theta) & \\
= & -\int_{0}^{\infty}\left[G_{+}^{\prime \prime}(\sigma, \eta) B_{i n}^{\prime}(\omega,-b)\right. \\
& -G_{+}^{\prime \prime}(\eta, \sigma) B_{i n}^{\prime}(\omega, c)-G_{+}^{\prime}(\sigma, \eta) B_{i n}^{\prime \prime}(\omega,-b) \\
& \left.+G_{+}^{\prime}(\eta, \sigma) B_{i n}^{\prime \prime}(\omega, c)\right] \omega J_{1}(\omega r \sin \theta) \mathrm{d} \omega \\
& -r^{-n+2 i-3}\left[(n+1) G_{n+1}^{-1 / 2}(\cos \theta) \csc \theta\right. \\
& \left.-2(i-1) G_{n}^{-1 / 2}(\cos \theta) \cot \theta\right],
\end{aligned}
$$

$$
\begin{aligned}
\gamma_{i n}^{(2)}( & r, \theta) \\
= & -\int_{0}^{\infty}\left[-G_{-}^{\prime}(\sigma, \eta) B_{i n}^{\prime}(\omega,-b)+G_{-}^{\prime}(\eta, \sigma) B_{i n}^{\prime}(\omega, c)\right. \\
& +G_{-}^{\prime \prime}(\sigma, \eta) B_{i n}^{\prime \prime}(\omega,-b) \\
& \left.-G_{-}^{\prime \prime}(\eta, \sigma) B_{i n}^{\prime \prime}(\omega, c)\right] \omega J_{0}(\omega r \sin \theta) \mathrm{d} \omega \\
& -r^{-n+2 i-3}\left[P_{n}\left(\cos \theta+2(i-1) G_{n}^{-1 / 2}(\cos \theta)\right],\right.
\end{aligned}
$$

where

$$
\begin{aligned}
& B_{1 n}^{\prime}(\omega, z)=-\frac{1}{n !}\left(\frac{\omega|z|}{z}\right)^{n-1} \mathrm{e}^{-\omega|z|}, \\
& B_{1 n}^{\prime \prime}(\omega, z)=-\frac{\omega^{n-1}}{n !}\left(\frac{|z|}{z}\right)^{n} \mathrm{e}^{-\omega|z|}, \\
& B_{2 n}^{\prime}(\omega, z) \\
& =-\frac{1}{n !}\left(\frac{\omega|z|}{z}\right)^{n-3}[(2 n-3) \omega|z|-n(n-2)] \mathrm{e}^{-\omega|z|},
\end{aligned}
$$

$B_{2 n}^{\prime \prime}(\omega, z)=-\frac{\omega^{n-3}}{n !}\left(\frac{|z|}{z}\right)^{n}[(2 n-3) \omega|z|$

$$
-(n-1)(n-3)] \mathrm{e}^{-\omega|z|},
$$

$G_{ \pm}^{\prime}(\mu, v)=\tau^{*} \mu v\left(\mu^{\prime} \pm \tau^{\prime} v^{\prime}\right)$,

$G_{ \pm}^{\prime \prime}(\mu, v)=\tau^{*}\left[v\left(\cosh \mu-\tau^{\prime} v^{\prime}\right) \pm \mu\left(\mu^{\prime}-\tau^{\prime} \cosh v\right)\right]$,

$\mu^{\prime}=\frac{\sinh \mu}{\mu}, \quad v^{\prime}=\frac{\sinh v}{v}, \quad \tau^{\prime}=\frac{\sinh \tau}{\tau}$,

$\tau^{*}=\frac{\tau}{\sinh ^{2} \tau-\tau^{2}}$,

$\sigma=\omega(r \cos \theta+b), \quad \eta=\omega(r \cos \theta-c)$,

$\tau=\omega(b+c)$.

\section{References}

Anderson, J.L., 1989. Colloid transport by interfacial forces. Annual Review of Fluid Mechanics 21, 61-99.

Anderson, J.L., Prieve, D.C., 1991. Diffusiophoresis caused by gradients of strongly adsorbing solutes. Langmuir 7, 403-406.

Anderson, J.L., Lowell, M.E., Prieve, D.C., 1982. Motion of a particle generated by chemical gradients. Part 1 . Non-electrolytes. Journal of Fluid Mechanics 117, 107-121.

Brenner, H., 1961. The slow motion of a sphere through a viscous fluid towards a plane surface. Chemical Engineering Science 16, 242-251.

Chang, Y.C., Keh, H.J., 2006. Slow motion of a slip spherical particle perpendicular to two plane walls. Journal of Fluids and Structures 22, 647-661.

Chen, P.Y., Keh, H.J., 2002. Diffusiophoresis of a colloidal sphere in nonelectrolyte gradients parallel to one or two plane walls. Chemical Engineering Science 57, 2885-2899.

Dukhin, S.S., Derjaguin, B.V., 1974. Electrokinetic phenomena. In: Matijevic, E. (Ed.), Surface and Colloid Science, vol. 7. Wiley, New York.

Ebel, J.P., Anderson, J.L., Prieve, D.C., 1988. Diffusiophoresis of latex particles in electrolyte gradients. Langmuir 4, 396-406.

Ganatos, P., Weinbaum, S., Pfeffer, R., 1980. A strong interaction theory for the creeping motion of a sphere between plane parallel boundaries. Part 1. Perpendicular motion. Journal of Fluid Mechanics 99, 739-753.

Hao, Y., Haber, S., 1998. Electrophoretic motion of a charged spherical particle normal to a planar dielectric wall. International Journal of Multiphase Flow 24, 793-824 
Happel, J., Brenner, H., 1983. Low Reynolds Number Hydrodynamics. Nijhoff, Dordrecht, The Netherlands.

Hsieh, T.H., Keh, H.J., 2007. Boundary effects on electrophoresis of a colloidal cylinder with a nonuniform zeta potential distribution. Journal of Colloid and Interface Science 315, 343-354.

Keh, H.J., Anderson, J.L., 1985. Boundary effects on electrophoretic motion of colloidal spheres. Journal of Fluid Mechanics 183, 458-475.

Keh, H.J., Chen, S.B., 1988. Electrophoresis of a colloidal sphere parallel to a dielectric plane. Journal of Fluid Mechanics 194, 377-390.

Keh, H.J., Chiou, J.Y., 1996. Electrophoresis of a colloidal sphere in a circular cylindrical pore. A.I.Ch.E. Journal 42, 1397-1406.

Keh, H.J., Hsu, Y.S., 2006. Diffusiophoresis of a colloidal sphere in nonelectrolyte gradients in a circular cylindrical pore. Chemical Engineering Science 61, 3550-3563.

Keh, H.J., Jan, J.S., 1996. Boundary effects on diffusiophoresis and electrophoresis: motion of a colloidal sphere normal to a plane wall. Journal of Colloid and Interface Science 183, 458-475.

Keh, H.J., Lien, L.C., 1991. Electrophoresis of a colloidal sphere along the axis of a circular orifice or a circular disk. Journal of Fluid Mechanics $224,305-333$
Keh, H.J., Luo, S.C., 1995. Particle interactions in diffusiophoresis in nonelectrolyte gradients. Physics of Fluids 7, 2122-2131.

Loewenberg, M., Davis, R.H., 1995. Near-contact electrophoretic particle motion. Journal of Fluid Mechanics 288, 103-122.

Maude, A.D., 1961. End effects in a falling-sphere viscometer. British Journal of Applied Physics 12, 293-295.

Morrison, F.A., Stukel, J.J., 1970. Electrophoresis of an insulating sphere normal to a conducting plane. Journal of Colloid and Interface Science 33, 88-93.

O'Brien, R.W., 1983. The solution of the electrokinetic equations for colloidal particles with thin double layers. Journal of Colloid and Interface Science 92, 204-216.

Staffeld, P.O., Quinn, J.A., 1989. Diffusion-induced banding of colloid particles via diffusiophoresis. 2. Non-electrolytes. Journal of Colloid and Interface Science 130, 88-100.

Unni, H.N., Keh, H.J., Yang, C., 2007. Analysis of electrokinetic transport of a spherical particle in a microchannel. Electrophoresis 28, 658-664.

Yariv, E., Brenner, H., 2003. Near-contact electrophoretic motion of a sphere parallel to a plane wall. Journal of Fluid Mechanics 484, 85-111. 